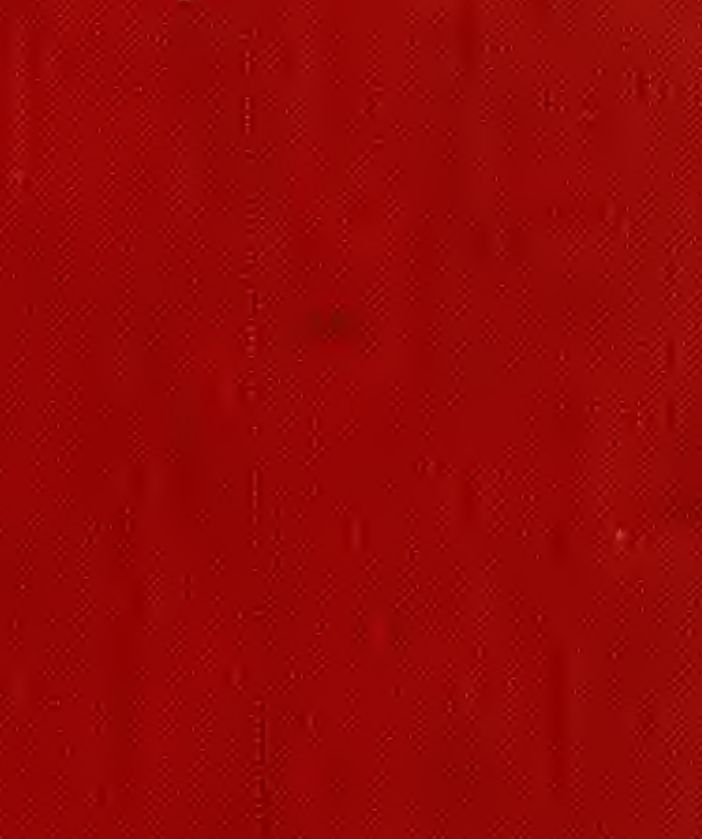



CRUSTACEA IIBRARY

SMITHSONIAN INST.

RETURN IO W-119 



\section{E. CHEVREUX}

Correspondant du Vuséum, à Bòne (Algérie)

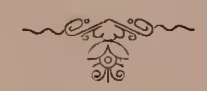

\section{REVISION DES}

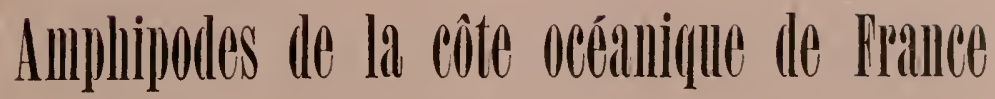

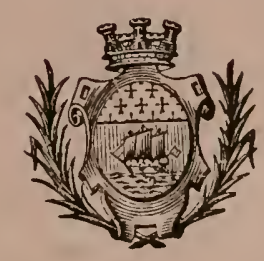

Extrait des Comptes rendus de

l'Association Française pour l'avancement des Sciences. Congrès de Nantes, 1898.

\section{PARIS}

SEGRÉTARIAT DE L'ASSOCIATION

Hôtel des Sociètés savantes

28, RUE SERPENTE, 28 



\section{E. CHEVREUX}

Correspondant du Muséum, à Bìne (Algérie)

\section{RÉVISION DES AMPHIPODES DE LA COTE OCÉANIQUE DE FRANCE}

Les Grustacés amphipodes de la côte océanique de France ont déjà fait l'objet de quelques publications. En 1887, M. Bonnier, dans son Catalogue des Crustacés malacostracés recueillis dans la baic de Concarneau ${ }^{1}$, énumère 66 espèces d'A mphipodes. A la même époque, je publiais un Catalogue des Crustacés amphipodes marins du SudOuest de la Bretagne ${ }^{2}$, comprenant 123 espèces, et, quelques mois plus tard, une liste supplémentaire de 17 Amphipodes ${ }^{3}$. Il y a lieu d'éliminer de ces listes quelques formes, qui ne diffèrent des espèces authentiques que par l'âge et le sexe, et de rectifier quelques erreur's de détermination.

D'autre part, les dragages effectués, au cours de ces dernières années, à bord de mes yachts Actif et Melita, m'ayant procuré un certain nombre d'espèces nouvelles pour la faune française, il m'a semblé utile de donner une liste définitive des Amphipodes rencontrés, jusqu’ici, sụr nos côtes de l’Océan.

1. Bulletin seient. du départ. đu Nord, 2e série, $\mathrm{X}^{e}$ année, 1887.

2. Bulletin de la Soc. Zonl. de France. XIIe vol., p. 288-340, pl. V.

3. Nouvelles espèces de Crustucés amphipodes du Sud-onest de la Bretagne, Assoc. française, congris de Toulouse, 1887. 
Dans le présent travail, j’ai considẻré comme appartenant ì la faune française toute l'étendue du plateau, constamment exploré par nos pêcheurs au chalut, qui descend en pente douce, depuis le littoral, jusqu'aux profondeurs de 180 ì $200 \mathrm{~m}$, et se termine par une falaise abrupte, à partir de laquelle commencent les grands fonds de lOcéan atlantique. On sait que ce plateau, large de plus de cent milles sous la latitude de Belle-Ile, décroît progressivement, pour n'avoir plus qu'une quinzaine de milles d'étendue, à la hauteur de l'embouchure de l'Adour.

J'ai tenu compte, dans la liste qui suit, des dragages du Moustique dans les parages des îles Glénans, en 1884, des recherches du baron J. de Guerne aux environs de Concarneau, en 1884, du catalogue publié par M. Bonnier en 188\%, et des dragages effectués par S. A. le

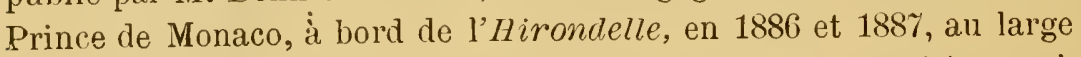
de la côte océanique de France, par des profondeurs inférieures à $200 \mathrm{~m}$. J'ai mentionné également une espèce nouvelle, draguée par l'aviso le Caudan', et un Amphipode appartenant à un genre nouveau, recueilli au voisinage de l'île d'Yeu par le bateau de pêche à vapeur anglais Britannic, et décrit par M. A. O. Walker. ${ }^{2}$ Enfin, les recherches de M. A. Dollfus dans le bassin d'Ar'cachon, à Guéthary, et ¿ Sl-Jean-de-Luz, m'ont permis de citer un certain nombre d'intéressantes espèces de nos côtes du Sud-Ouest.

\section{HYPERIDE}

1. Hyperia galba (Mont.) - Toute la côte, dans les Discoméduses (tihizostoma, Aurclia, Cyanea, Chrysaora).

2. Parathemisto oblivin (Kroyer) - Prise au filet entre deux eaux, pendant les dragages de la Melita (au large de Pen-March, de l'île d'Yeu, du phare de Contis, de l'embouchure de l'Adour), par 50 à $90 \mathrm{~m}$ au-dessous de la surface, et dans l'estomac d'un Germon pêché au large des Iles Glénans.

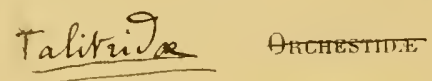

3. Talitrus locusta (Pallas) - Toute la côte, sur les plages de sable fin.

4. Talorchestia Deshayesi (Audouin) - Le Croisic.

5. T. brito Stebbing - Plage du Verdon (embouchure de la Gironde).

gammarallum (Tallan) niveau des pleines mers.

6. Orchestia tittorea (Hout:) - Toute la còte, sous les pierres, au-dessus du

1. Résultats scientifiques de la campagne du Caudan dans le golfe de Gascogne, aoul-septembre 1895. Fascicule III. Edriophthatmes par Jules Bonnier. Annales de l'Université de Lyon, Paris 1896.

2. On two newo species of $\mathrm{Amphipoda}$ Gammarider. Annals and Nag. of Nat. History, série fi, vol. X VIII, anù1 $18 \%$ ' 
m. 5 . 7.0 .4 Hetley. - Nantes 1 .

8. 0. mediterranea Costa - Belle-Ile, la Trinité de Crach, le Croisic - Sı-Jeande-Luz (Dollfus).

9. Hyale Nitssoni (Rathke) - Concarneau (Bonnier) - Belle-lle, le Croisic, Royan - Guéthary (Dollfus).

10. H. Prévnsti (MI.-Edw.) - Belle-Ile, le Croisic - St.Jean-de-Luz (Dollfus).

11. II. pontica Rathke 2 - Pontaillac, embouchure de la Gironde (Dollfus). hmidf 12 H. (Heller) - Guéthary, St-Jean-de-Luz (Dollfus).

\section{LYSIANASSID.F.}

13 Acidostoma obesum (Sp. Bate) - Iles Glénans, $23 \mathrm{~m}$; le Croisic, 11 m (Actif!

14. Ichnopus spinicornis Boeck - Sud-ouest de Belle-Ile, $130 \mathrm{~m}$ et $160 \mathrm{~m}$ (Melita).

15. Lysianax ceratinus Walker - Iles Glénans, $10 \mathrm{~m}$ et $23 \mathrm{~m}$ (Actif) - Betle-Ile, 10 m (Hirondelle). - Le Croisic, marée basse.

16. L. Costa (M. Edw.) - Rade de Brest, $30 \mathrm{~m}$ (Melita) - Concarneau (Bonnier) - Iles Glénans, $60 \mathrm{~m}$; baie de Quiberon, le Croisic, 8 à $10^{\mathrm{m}}$ (Actif).

17. Socarnes erythrophthalmus Robertson - lles Glénans, baie de Quiberon, Piriac, le Croisic, 0 à $15 \mathrm{~m}$ (Actif).

18. Perrierella audoniniana (Sp. Bate)-Rade de Brest, $30 \mathrm{~m}$ (Iclita) - Iles Glénóns, Concarneau, le Croisic, 15 à $23 \mathrm{~m}$ (Actif).

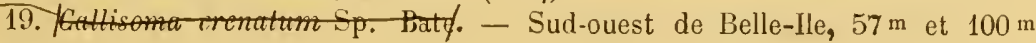
(Mclita).

20. Hippomedon denticulatus (Sp. Bate) - Concarneau, 45 à $50 \mathrm{~m}$ (Bonnier) - Le Groisic, 5 à $10 \mathrm{~m}$ (Actif).

ilis (cots) 21. Orchomene - O. Rade de Brest, 30 m (IIelita) - Concarneau (Bonnier) - Baie de Quiberon, le Croisic, 0 à $6 \mathrm{~m}$ (Actif).

22. Orchomenclla nana (Kröyer) - Rade de Brest, nasse, $8 \mathrm{~m}$ (Melita) - Iles

Giénans, Port-Navalo, le Croisic, nasse, 10 à 19 m (Actif) - Arcachon (Dollfus).

23. Nannonyx Goësi (Boeck) - Le Croisic, marée basse.

24. Tryphosites longipes (Sp. Bate) - Le Croisic, $10 \mathrm{~m}$ et $15 \mathrm{~m}$ (Actif) - Sudouest de Belle-Ile, 136 et $180 \mathrm{~m}$ (Hirondelle).

25. Centromedon crenulatum nov. $\mathrm{sp}^{3}$. - Sud-ouest de Belle.Ile, $180 \mathrm{~m}$ (IIrondelle).

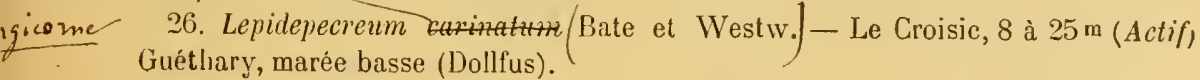

27. L. clypeatum Chevreux - Sud-ouest de Belle-Ile, $180 \mathrm{~m}$ (Hirundelle).

28. Podoprion Bolivari Chevreux - Saint-Sébastien (Bolivar) 4.

1. Une douzaine d'exemplaires trourés, en compagnie de nombreux Niphrirgus puteanus, au fond d'un puits que l'on vidait pour le nettoyer.

2. H. Lubbockicna (Sp. Bate).

3. Les espéces nouvelles, citées dans cette liste, sont décrites et figurées dans un mémoire, en cours d'impression à Monaco, intitulé : Crustcicés Amphipodes p'ovenant des Campagnes du Yacht l'Hirondelle, 1885-1888.

4. Cette espèce, décrite d'aprés des exemplaires recueillis par la Melitu dans la haie de Vigo, a étẻ retrouvée depuis, au voisinage de la frontière de France, par le Professeur Bolivar. Je la cite ici jour mémoire, persuadé qu'elle sera capturée tòt ou tard sur nos côtes du Sud-Ouest. 


\section{PONTOPOREID.E}

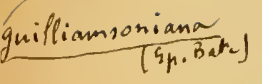

29. Bathyporcia partegiet G. (1. Dar = Le Croisic, $10 \mathrm{~m}$ (Actif),

30. B. pclagica Sp. Bate - Le Croisic, $10^{\mathrm{m}}$ (1ctif) - Pontaillar, marée bàsse. (Dollfus).

31. B. Rubértsoni Sp. Bate -- Le Croisic, 5 à $6^{\text {na }}$ (Actif).

32. Hanstorius arenarius (Slabber) - Le Croisic, le Pouliguen, marée basse.

33. Urolhoc marina (Sp. Bate) - Concarneau (Bonnier) - Iles Glénans, $19^{\mathrm{m}}$ (Monstiquc) - Le Croisic, 6m (Actif) - Sud-ôuest de Belle-Ile, $180 \mathrm{~m}$ (IIrondelle).

31. U. brevicomis Sp. Bate - Le Croisic. le Pouliguen, 0 à 5 m (Actif).

35. U. clegans Sp. Bate - Sud-ouest de Belle-Ile, $180 \mathrm{~m}$ (Hirondelle).

36. U. pulchella (Costa) - Le Croisic, 5 à $6 \mathrm{~m}$ (Actif).

37. U. Grımaldii Chevreux - S'-Jean-de-Luz, 5 à $6 \mathrm{~m}$ (Dolfus).

38. Argissa hamatipes (Norman) - Sud-ouest de Belle-Ile, $160 \mathrm{~m}$ (IItita).

\section{PhOXOCEPHALID.E}

\section{Metaptionus}

39. Fultoni(Th. Scott/-- Le Croisic, 10 à $20 \mathrm{~m}$ (Acttf).

40. Paraphoxus maculatus (Chevreux) - Sud-ouest de Belle-Ile, 180 m (IIrondelle).

41. Metaphoxus pectinatus (WValker) - Rade de Brest, 30 m (Mclita) - Iles Glènans, $60 \mathrm{~m}$; Concarneau, $18 \mathrm{~m}$; le Croisic, 15 à $20 \mathrm{~m}$; ouest de l'île d'Yeu, $80 \mathrm{~m}$ (Actif) - Belle-Ile, $19 \mathrm{~m}$ (Hirondellc).

42. II urpinia neglecta G. 0. Sars - Concarneau, $19 \mathrm{~m}$ (Actif) - Belle-Ile, $19 \mathrm{~m}$ (Hirondelle).

43. 11. pectinata G. 0. Sars - Concarneau, baie de Quiberon, le Croisic, 10 à $20^{\mathrm{m}}$ (Actif) - Saint Jean-de-Luz, (6 m Dollfus).

44. II. crenulata Boeck - hade de Brest, $30^{\text {m }}$ (Mclita) - Le Croisic, 10 à $18^{\text {m }}$ (Actif) - Belle-Ile, $19 \mathrm{~m}$ (Itirondelle).

\section{AMPELISCID.E}

45. Ampelisca typica (Sp. Bate) - Concarneau (Bonnier) - Iles Glénans, $60 \mathrm{~m}$; Concarneau, le Croisic, 15 à $19^{\mathrm{m}}$ (Actif).

46. A. tonuicornis Lillj. = Concarneau, le Croisic, 15 à $19 \mathrm{~m}$ (Actif) - Belle-Ile, $19 \mathrm{~m}$ (IIrondellc).

47. H. Assimitis Boeck - Iles Glénans, $60 \mathrm{~m}$; le Croisic, $16 \mathrm{~m}$ (Actif) - Nordouest de Saint-Jean-de-Luz, $120 \mathrm{~m}$ (Mctita).

$=$ 48. A. diadema (Costa) - Iles Glènans, 50 à $60 \mathrm{~m}$ (de Guerne) - Le Croisic, 10 à $15 \mathrm{~m}$ (Actif) - Belle-Ile, $19 \mathrm{~m}$ (IIirondelle).

49. A. brevicornis (Costa)' - Iles Glènans, le Croisic, 0 à $30 \mathrm{~m}$ (Actif) - BelleIle, $19 \mathrm{~m}$; Sud-ouest de Belle-Ile, $180 \mathrm{~m}$ (Hirondclle) - Arcachon, Saint-Jean-deLuz, 6 à $8 \mathrm{~m}$ (Dollfus).

50. A. Sarsi Chevreux - Concarneau, le Croisic, 15 à $19 \mathrm{~m}$ (Actif) - Saint.Jeande Luz, 5 à $6 \mathrm{~m}$ (Dollfus).

\section{A. levigata Lillj.}


51. A. spinimana Cherreux - Le Croisic, 15 à $20 \mathrm{~m}$ (Actif) - Saint-Jean-deLuz, $10 \mathrm{~m}$ (Uelita).

52. A. spinipes Boeck - Iles Glénans (Moustique) - Concarneau, Lorient, le Croisic, l'île d'Yeu, 15 à $60 \mathrm{~m}$ (Actif) - Belle-1le, 19m; Sud-ouest de Belle-Ile, 136m et $166 \mathrm{~m}$ (IIrondclle) - Saint-Jean-de-Luz, $120 \mathrm{~m}$ (IIelita).

53. A. cequicornis Bruz. - lles Glénans, Gom (Actif).

54. A. rubella Costa - Saint-Jean-de-Luz, $8 \mathrm{~m}$ (Mrlita).

55. Haploops tubicola Lillj. - Iles Glénans (Moustique) - Concarneau (de Guerne, Bonnier) - Concarneau, le Croisic, 15 à $20 \mathrm{~m}$ (Actif) - Belle-Ile, $19 \mathrm{~m}$ (Ilirondelle).

\section{StegociephaLid.e}

56. Stegocephaloides christianiensis (Boeck) - Sud-ouest de Belle-Ile, $180 \mathrm{~m}$ (Hirondelle) - Sud-ouest de Belle-Ile, $150 \mathrm{~m}$ (Melita).

\section{AMPHILOCHID.E}

57. Amphilochus manudens Sp. Bate. - Sud-ouest de Belle-Ile, $180 \mathrm{~m}$ (Hirondelle) - Le Croisic, $10 \mathrm{~m}$ (Actif).

58. A. neapolitanus Della Valle - Goncarneau, 19 m (Actif) - Guéthary, marée basse (Dollfus).

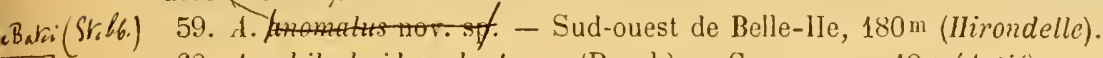

60. Amphilochoides odontonyx (Boeck) - Concarueau, 19m (Actif).

61. A. longimanus (Cherreux) - Sud-ouest de Belle-Ile, $180 \mathrm{~m}$ (IIrondelle).

62. Gitana Sarsi Boeck - Baie de Quiberon, le Croisic, 7 à 14 m (Actif) - Sudouest de Belle-1le, 180 m (IIirondelle).

riomi Catta 63. Pcltocoxa (tammoniensis (Stebbing) - Le Croisic, 0 à $6 \mathrm{~m}$, roches (Actif).

\section{STENothoIde}

64. Stenothoe marinı (Sp. Bate) - Concarneau, $20 \mathrm{~m}$ (Bonnier) - Concarneau, baie de Quiberon, le Croisic, 11 à $19 \mathrm{~m}$ (Act,if) - Arcachon, Saint-Jean-de-Luz, 0 à $5 \mathrm{~m}$ (Dollfus).

65. S. monoculoides (Mont.) - Concarneau, 0 à $25^{\mathrm{m}}$ (Bonnier) - Le Croisic, marée basse - Arcachon, Guéthary, Saint-Jean-de-Luz, marée basse (Dollfus).

66. Metopa rubrovittata G. 0. Sars - Sud-ouest de Belle-Ile, $180 \mathrm{~m}$ (Hiron(delle).

67. Cressa dubia (Sp. Bate) - Le Croisic, $6 \mathrm{~m}$, roches (Actif).

\section{LEUCOTHOIDE}

68. Leucothoc spinicarpa (Abildg.) - Concarneau (de Guerne, Bonnier) - Rade de Brest, $30 \mathrm{~m}$; ouest de l'île d'Yeu, $100 \mathrm{~m}$ (Melita) - Iles Glénans, Concarneau, baie de Quiberon, le Croisic, 10 ì $20 \mathrm{~m}$ (Actif) - Belle -Ile, $10 \mathrm{~m}$ (Hirondelle).

69. L. incisa Robertson - Iles Glénans, 50 à $60 \mathrm{~m}$ (de Guerne) - Belle-Ile, $19 \mathrm{~m}$ (Hirondelle) - Le Croisic, 0 à $15 \mathrm{~m}$ (Actif) - Arcachon, Saint-Jean-de-Luz, 5 à $6 \mathrm{~m}$ (Dollfus).

OEDICERID.E

79. Monoculodes carinatus Sp. Bate - Concarneau, $50 \mathrm{~m}$ (Bonnier) - Lorient, le Croisic, 6 à $23 \mathrm{~m}$ (Actif). 
71. M. gibbosus Chevreux - Sud-ouest de Belle-Ile, $180 \mathrm{~m}$ (Hirondelle).

72. Perioculodes longimanus (Bate et Westw.) - Rade de Brest, $30 \mathrm{~m}$ (Melita) Le Croisic, $6 \mathrm{~m}$ (Actif) - Sud-ouest de Belle-Ile, $180 \mathrm{~m}$ (Itirondelle) - Arcachon, Saint-Jean-de-Luz, 5 à $6 \mathrm{~m}$ (Dollfus).

73. Pontocrates arenarius (Sp. Bate) - Le Croisic, 5 à $10 \mathrm{~m}$ (Actif) - SaintJean-de Luz, 5 à $6 \mathrm{~m}$ (Dollfus).

74. P. altamarinus (Sp. Bate) - Concarneau, 50m (Bonnier) - Le Croisic, $10 \mathrm{~m}$ (Actif).

75. Synchelidium haplocheles (Grube) - Rade de Brest, $30 \mathrm{~m}$ (IIClita) - Concarneau, $19 \mathrm{~m}$ (dctif) - Pontaillac (Dollfus).

76. Halimedon Mülleri Boeck - Sud-ouest de Belle-Ile, $180 \mathrm{~m}$ (Hirondelle).

77. H. rectirostris Della Valle - Rade de Brest, $30 \mathrm{~m}$ (Melita) - Concarneau, $15 \mathrm{~m}$ (Actif) - Belle Ile, $19 \mathrm{~m}$ (Hirondelle).

\section{Paramphithold.e}

78. Stenopleustes nodifer G. O. Sars - Sud-ouest de Belle-Ile, $180 \mathrm{~m}$ (IIirondelle).

\section{EPLMER:D症}

79. Epimeria cornigera (Fabr.) - Sud-ouest de Belle-1le, 155 à $180 \mathrm{~m}$ (IIirondelle).

\section{Pardaliscid $\approx$}

80. Halicoides anomala Walker - Ile d'Yeu, 31 à $58^{m}$ (chalutier à vapeur anglais Britannic).

\section{IPHIMEDIDE}

81. Iphimedia obesa Rathlse - Belle-Ile, $10^{\mathrm{m}}$ (IIrondelle) - Belle-Ile, le Croisic, 10 à 50 m (Actif).

82. I. minuta G 0. Sars - Le Croisic, 5 à $6^{\text {m }}$ (Actif).

83. Iphimediopsis Eblance (Sp. Bate) - Iies Glénans, le Croisic, 6 à $10 \mathrm{~m}$, roches (Actif).

8'. Guernea coalita (Norman) - Le Croisic, 0 à 10 m (Actif) - Guéthary, SaiutJean-de-Luz (Dollfus).

\section{LAPHYSTIDE}

85. Laphystius Sturionis Kröyer - Sud-ouest de Belle-Ile, 80 w (sur un Maia squinado).

\section{EUSIRID}

86. Eusirus longipes Boeck - Iles Glénans, 6 a 8 in (Actif) - Sud-ouest de Belle-Ile, $166 \mathrm{~m}$ et $180 \mathrm{~m}$ (Hirondelle).

87. Ensiroides Della Vallei nov. nom. ${ }^{1}$ - Guéthary, Saint-Jean-de-Luz, marée basse (Dollfus).

- 1. Cette espèce a été décrite par le professeur Della Valle, sous le non d'Eusiroides Ccesaris Stebbing, l'après des exemplaires provenant du golfe de Naples. J'ar ou occasion de la retrouver en plusieurs points de la côte méditerranéenne de France (Port-Vendres, Saint-Raphaël, Cannes, Antibes), et à Cadix. Elle est à mon avis, bien distincte des trois formes d'Eusiroides draguées par le Challenger dans l'hemisphère sud, et je propose de la nommer Eusiroides Della Vallei. 


\section{Calliopide}

83. Apherusa bispinosa (Sp. Bate) - Iles Glénans, Groix, baie de Quiberon, le Croisic, 0 à $15 \mathrm{~m}$ (Actif) - Belle-Ile, 10m ; sud de Belle-Ile, 63m (Hirondelle) Arcachon (Dỏllus).

89. A. Jurinci (M.-Edw.) - Douarnenez, Concarneau. le Groisic, Saint-Jeande-Luz, marée basse - Guéthary (Dollfus).

90. A. cirrus (Sp. Bate) - Le Croisic, marée basse.

91. A. tridentata (Bruz.) - Sud-ouest de Belle-Ile, 160m (Nelita).

92. Calliopins Rathlici (Zaadach) - Douarnenez, marée basse.

\section{ATYLIDE}

93. Paratylus Swammerdami (M.-Edw.) - Concarneau (Bonnier) - Le Croisic, le Pouliguen, 0 à $10 \mathrm{~m}$ (Actif) - Royan, Arcachon, Saint-Jean-de-Luz (Dollfus)

94. P. uncinatus (G. O. Sars) - Le Croisic, 6 à $8 \mathrm{~m}$ (Actif).

95. P. vedlomensis (Sp. Bate) -C Concarneau 15m (Bonnier) - Concarneau, 19m (Actif).

96. P. guttatus (Costa) - Concarneau, baie de Quiberon, le Croisic, 6 à 10 (Actif) - Pontaillac, Arcachon (Dollfus) - Saint-Jean-de-Luz, $8^{m}$ (Mctita).

97. Dexamine spinosa (Mont.) Rade de Brest, 28m (Melita) - Hes Glénans (Moustique) - Concarneau (Bonnier) - Belle-Ile, 10 (Hirondelle) - Douarnenez, le Croisic, marée hasse - Arcachon, Guéthary, Saint-Jean-de-Luz (Dollfus).

98. D. Thea Boeck - Le Croisic, marée basse - Guéthary, Hendaye (Dollfus). 99. D. spiniventris (Costa) - Guéthary, Saint-Jeau-de-Luz, marée basse (Dolllus).

100. Tritceta yibbosa (Sp. Bate) - Concarneau (Bonnier) - Le Croisic, 0 à $6^{\mathrm{m}}$, roches, dans les Eponges (Actif).

\section{Gammarid.e}

101. Mclphidippclla macera (Norman) - Ouest de l'ỉe d'Yeu, 75m (Actif).

102. Homari (Fabr.) - Concarneau (Bonnier) - Le Croisic, le Pouliguen, marèe basse.

103. A angulosa (Rathke) - Le Croisic, le Pouliguen, marée, dans les Algues. 104. Gammarus marinus (Leach) - Concarneau (Bonnier) - Groix, le Croisic, Royan, marée basse.

105. G. locusta (Linné) - Iles Glénans (Moustique) - Concarneau (Bonnier) Le Croisic, 0 à $\gamma_{m}^{m}$ (Actif) - Arcachon, Saint-Jean-de-Luz (Dollfus).

106. G. Ducbeni Lillj - Au bord de la Loire, depuis Saint-Nazaire jusqu'en amont de Nantes - Prairie de Mauves (L. Bureau).

107. Gammarella brevicaudata (M -Edw.) - Iles Glénans (de Guerne) - Concarneau (Bonnier) - lles Glènans, le Croisic, 0 à $8^{\mathrm{m}}$ (Actif) - Arcachon, Guéthary, Saint-Jean-de-Luz (Dollfus).

108. Velita palmata (Mont.) - Concarueau (Bonnier) - Le Croisic, la hochelle, narée basse - Arcachon, Guéthary, Saint-Jean-de-Luz (Dolllus).

109. M. obtusata (Mont.) - Concarneau, $30^{\mathrm{m}}$ (Bonnier) - Le Croisic, $10^{\mathrm{m}}(\mathrm{Ac}$ - 
tif) - Sud-ouest de Belle-Ile, 180m (Hirondelle) — Sud-ouest de Belle-İle, 160m (IIelita) - Arcachon (Dollius).

110. M. gladiosa Sp. Bate - Iles Glénans, 29m (Moustique) - Concarneau, 10m (Bonnier) - Groix, le Croisic, 10 ${ }^{\mathrm{m}}$ (Actif) - Belle-Ile, 10m; Sud-ouest de BelleIle, $160 \mathrm{~m}$ (Hirondelle).

111. Mara Othonis (M.-Edw.) - Rade de Brest, 23m; Sud-ouest de Belle-Ile, $160 \mathrm{~m}$ (Mctita) - Concarneau, (de Guerne) - Belle-Ile. 19m; Sud-ouest de BelleIle, $166^{\mathrm{m}}$ et $180^{\mathrm{m}}$ (Hirondelle) - Groix, le Croisic, île d'Yeu, 10 à 75m (Actit).

112. M. grossimana (Mont.) - Concarneau, 10m (Bonnier) - Le Croisic, marée basse.

Cerasous

113. 1. semiserrata (Sp. Bate) - Belle-Ile, 10m (Hirondelle) - Baie de Quiberon, $8 \mathrm{~m}$ (Actif).

114. H. Batei Vormat - Baie de Quiberon, le Croisic, 5 à $27^{\mathrm{n}}$ (Actif) - Sudouest de Belle-Ile, $180 \mathrm{~m}$ (Hirondelle).

115. Megaluropus agilis Norman - Le Croisic, $8 \mathrm{~m}$ (Actif).

116. Elasmopus rapax (Costa) - Concarneau (Bonnier) - Iles Glénans, $8 \mathrm{~m}$ (Actrf) - Belle-Ile, le Croisic, 15 à $80 \mathrm{~m}$, sur les Maia - Guéthary, Saint-Jeande-Luz (Dolllus).

117. Cheirocratus Sundcvalli (Rathke) - Rade de Brest, $30 \mathrm{~m}$ (Melita) - BelleIle, $10 \mathrm{~m}$ (Hirondelle) - Baie de Quiberon, Piriac, le Croisic, 0 à $8 \mathrm{~m}$ (Actif).

118. G. intermedius G. O. Sars - Le Croisic, $10 \mathrm{~m}$ (Actif) - Arcachon (Dollfus).

119. Lilljcborgia pallida (Sp. Bate) - Concarneau (Bonnier) - Sud-ouest de Belle-Ile, $180 \mathrm{~m}$ (IIirondclle) - Sud-ouest de Belle-Ile, $100 \mathrm{~m}$ (IIclita).

120. L. fissicornis (M. Sars) - Sud-ouest de Belle-Ile, $180^{\mathrm{m}}$ (Hirondelle).
121. Le picta(Normant-Belle-Ile, $10 \mathrm{~m}$ (Hirondelle) - Baie de Quiberon, 8 et $27 \mathrm{~m}$ (Actif) - Saint-Jean-de-Luz, 5 à $6 \mathrm{~m}$ (Dolllus).

122. Kinahani Sp. Bate - Baie de Quiberon, $14 \mathrm{~m}$ (Actif).

123. Isiea Montagui M.-Edw. - Concarneau (Bonnier) - Port-Navalo, le Croi sic, commensal de Maia squinado et de Homarus vulgaris.

\section{Photid.e}

124. Microdeutopus gryllotalpa Costa - Le Croisic, le Pouliguen, dans les marais salants et dans les pares à huîtres - Arcachon (Dollfus).

125. M. versiculatus (Sp. Bate)-Concarneau (Bonnier) - Baie de Quiberon 7 à $14 \mathrm{~m}$ (Actif) - Arcachon, Saint-Jean-de-Luz (Dollfus).

126. M. anomalus (Rathke) - Sud-ouest de Belle-Ile, $100 \mathrm{~m}$ (Melita).

127. M. danmoniensis Sp. Bate - Concarneau, $19 \mathrm{~m}$ (Actif).

128. M. Statıonis Della Valle - Arcachon (Dollfus).

129. Stimy chelifera (Sp. Bate) - Ile Dumet (Actif) - Guéthary (Dollius).

430. \$. armata (Chevreux) - Le Croisic, $18 \mathrm{~m}$ (Actif).

131. Aora gracilis (Sp. Bate) - Concarneau (Bonnier) -- Concarneau, le Croisic, 10 à $19 \mathrm{~m}$ (dctif) - Arcachon, Saint-Jean-de-Luz (Dolllus).

- 132. Lembos Wcbsteri Sp. Bate - Iles Glénans (de Guerne) - Concarneau (Bonnier) - Le Croisic, marée hasse - Guéthary (Dollfus)

133 L. longipes (Lillj.) - Iles Glénans, $60 \mathrm{~m}$ (Actif).

131. Leptocheirus guttatus (Grube) - Concarneau, Lorient, baie de Quiberon, le Groisic, 10 à $50 \mathrm{~m}$ (Actif) - Belle-Ile, $10 \mathrm{~m}$ (Hirondelle). 
inalus hruman 135. L. Iles Glénans, Lorient, le Croisic, 0 à $20 \mathrm{~m}$ (Actif) - Belle-Ile, $10 \mathrm{~m}$ et $19^{\mathrm{m}}$ (IIirondelle).

136. L. hirsutimanus (Sp. Bate) - Le Croisic, $18 \mathrm{~m}$ (Actif) - Sud-ouest de Belle-Ile, $180 \mathrm{~m}$ (IIirondelle).

13\%. maculata (Johnston) - Concarneau (de Guerne, Bonnier) - Sud-Ouest de Belle-Ile, $155 \mathrm{~m}$ (IIrondelle) - Belle-lle, le Croisic, 10 à $60 \mathrm{~m}$

(Actif) - Royan, Arcachon, Guéthary (Dollfus).

138. Gr. melanops G. 0. Sars - Lorient. $20 \mathrm{~m}$ (Actif).

139. Megamphopus cornutus Norman - Iles Glénans, baie de Quiberon, le Croisic, 10 à $60 \mathrm{~m}$ (Actif) - Sud-ouest de Belle-Ile, $180 \mathrm{~m}$ (IIironilelle).

140. Aticroprotopus maculatus Norman - Mesquer, le Youliguen, 5 à $10^{m}$ (Actif)

- Pontaillac, Arcachon, Saint-Jean-de-Luz (Dollfus).

141. M. longimanus Cherreux - Le Croisic, marée basse.

142. Photis longicaudala (Bate et Westw.) - Concarneau (Bonnier) - Concarneau, le Croisic, 10 à 20 m (Actif) - Belle-Ile, $19^{\mathrm{m}}$; Sud-ouest de Belle-lle, $180^{\mathrm{m}}$ (IIrondelle) - Arcachon (Dollfus) - Saint-Jean-de-Luz, $120 \mathrm{~m}$ (IItitr).

143. Podoceropsis Sophice Boeck - Concarneau, le Croisic, 19 à $23 \mathrm{~m}$; île d'Yeu, $75 \mathrm{~m}$ (Actif).

$=$ 144. P. . Butata Sp. Bete-Sud de Belle-Ile, $60 \mathrm{~m}$, sur un Maia squinado.

\section{PODOCERID}

W 145. Amphithoe rubricata (Mont.) - Douarnenez, Concarneau, baie de Quiberon, le Croisic, le Pouliguen, 0 à $20 \mathrm{~m}$ (Actif) - Concarneau (de Guerne, Bionnier) - Belle-lle, $10 \mathrm{~m}$ (Hirondclle).

146. A. Vaillanti Lucas - Guéthary, Saint-Jean-de-Luz, marée basse (Dodfus).

147. A.cunicutus Stebbing - Le Croisic, marée basse."

148. Pleonexes gammuroiles Sp. Bate - Concarneau (Bonnier) - Le Croisic marée basse - Areachon, Guéthary, Saint-Jean-de-Luz (Dollfus).

149. Sunamphithne pelagica (M. Edw.)2 - Douarnenez, Conearneau, le Croisie, warée basse - Guéthary, Saint-Jean-de-Luz (Dollfus).

150. Portocrtos falcatus (Mont.) - Douarnenez, Mesquer, le Croisic, marée

assa basse, et sur les bouées (Actil) - Concarneau (Bonnier) - Saint-Jean-de-Luz (Dolllius).

151. Pै pusillus G. O. Sars - Belle-1le, le Croisic, 18 à $20 \mathrm{~m}$ (Actif).

Tentax Gam 152. P. Hertmani Walked - Sud de Belle-1le, $60 \mathrm{~m}$, sur un Maia squinado.

153. $V_{\text {; }}$ ocius Sp. Bate - Le Croisic, dans des Eponges - Guéthary (Dollfus).

154. Pu cumbrensis(Stebbing et Robertson) - Baie de Quiberon, $10 \mathrm{~m}$ (Actif).

155. Le Croisic, le Pouliguen, marée basse - Saint-

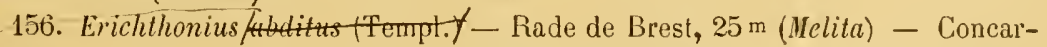
ifiemis (Pangau, le Croisic, 10 à $20 \mathrm{~m}$ (Actip) - Concarneau (Bonnier) - Sud de Belle-lle, $63 \mathrm{~m}$ (IIirondelle) - Au large d'Arcachon, $80^{\mathrm{m}}$; Saint-Jean-de-Luz, $6^{\mathrm{m}}$ (Dollfus).

157. E. difformis M.-Edw. - Concarneau (Bonnier) - Le Croisic, marée basse

- Saint-Jean-de-Luz, $8 \mathrm{~m}$ (Melita).

1. Cette petite forme, dont je n'ai rencontré qu'un seul exemplaire, est peut-être l'état jeune d'une espèce voisine.

2. Sunamphitho conformata sp. Bate. 


\section{COROpHID.e}

158. Siphonocetes Collelti Boeck - Concarneau, baie de Quiberon, le Croisic, le Pouliguen, 6 à $19 \mathrm{~m}$ (Actif) - Belle-Ile, 155 à $180 \mathrm{~m}$ (Hirondelic) - Sud-ouest de Belle-Ile, $160 \mathrm{~m}$ IIclita) - Saint-Jean-de-Luz, 5 à $6 \mathrm{~m}$ (Dolltus).

159. Corophium (Lines - Le Croisic, le Pouliguen, dans les marais salants - Saint-Jean-de-Luz (Dollfus).

160. Á. acherusicum Costa - Concarneau (Bonnier) - Baie de Quiberon, le Croisic, 0 à 6 m (Actif) - Baie de l'Aiguillon, Royan, Pontaillac, Arcachon (Dollfus).

161. Unciola crenatipalmata (Sp. Bate) - Belle-Ile, $19 \mathrm{~m}$; Sud-ouest de BelleIle, 136 à $166 \mathrm{~m}$ (IIrondclle) - Ouest de Belle-Ile, 130 m; Saint-Jean-de Luz. $8 \mathrm{~m}$ (Mclita) - Le Croisic, 10 à $18 \mathrm{~m}$ (Actif).

11i2. U. planipes Norman - Iles Glénans, 50 à $60 \mathrm{~m}$ (de Gucrne) - Sud ouest de Belle-Ile, $180 \mathrm{~m}$ (Ilirondelle) - Ouest de l'lle d'Yeu, $50^{\mathrm{m}}$ el $75 \mathrm{~m}$ (Actif).

163. U. incerta Bonnier - Au large de l'embouchure de lin Gironde, $180 \mathrm{~m}$ (Candan).

161. Scba urmata (Chevreux) - Sud-ouest de Belle-Ile, $180 \mathrm{~m}$ (IIrondelle).

\section{ChéLuride}

165. Chclura tercbrans Philippi - Concarneau (Bonnier).

\section{DULICHID.E}

166. Platophum Darwini (Sp. Bate) - Concarneau (de Guerne) - Le Croisic, marée basse - Saint-Jean-de-Luz (Dollfus).

\section{Colomastide}

167. Colomastix pusilla Grube - Concarneau, le Croisic, dans des Eponges, 0 à $19 \mathrm{~m}$ (Actif).

\section{Gaprellide}

168. Phtisica marina Slabber - Concarneau (Bonnier) - Belle-Ile, 19m (Hirondelle) - Baie de Quiberon, le Croisic, 0 à $14^{\mathrm{m}}$ (Actif) - Sud-ouest de Belle-Ile, $100 \mathrm{~m}$ (Melita) - Arcachon (Dollfus).

169. Prof phasma (Mont.) - Lorient, le Croisic, 0 a $19 \mathrm{~m}$ (Actif) - SaintJean-de-Lu\% (Dolllus).

170. Parvipalpus capillaceus (Chevreux) - Concarneau, le Croisic, 6 à $20 \mathrm{~m}$ ( $\mathrm{Ac}$ tif).

171. Pariambus typicus (Kröyer) - Belle-Iie, $60 \mathrm{~m}$, sur les Maia squinado - Le Croisic, 10' sur Asteracanthion rubens (Actil) - Arcachon (1)ollfus) - Saint-Jeande-Luz, $10^{\mathrm{m}}$, sur Asteracanthion rubens (Nelita).

172. Caprelı́a acutifrons Desmarest - Concarneau (Bonnier) - Le Ciroisic, marée basse - Royan, Arcachon, Guéthary, Saint-Jean-de-Luz (Dollfus).

- 173. C. cequilibra Say - Port militaire de Lorient (Hirondelle) - Arcachou, Saint-Jean-de-Luz (Doll'us).

174. C. acauthifera Leach - Douarnenez, le Croisic, 0 i $6^{\mathrm{m}}$ (Actif) - Concarneau (Bonnier) - Belle-Ile, I0m (Ilirondelle) - Guéthary, Saint-Jean-de-Luz (Dollfus). 
175. C. tuberculata Guérin - Concarneau (Bonnier) - Le Croisic, marée basse 176. C. fretensis Stebbing - Le Croisic, 6m, roches (Actif).

A ces 176 espèces, on peut ajouter trois formes d'eau douce, habitant au voisinage du littoral; ce sont:

Gammarus pulex Linné - Commun partout, au bord des ruisseaux et des étangs. de-Luz.

Cr.Ch. Niphargus Le Croisic, dans les puits.

L'étude de la répartition géographique des espèces mentionnèes ci-dessus présente un certain intérèt. Quelques formes méditerranéennes se trouvent sur nos côtes du Sud-Ouest, et ne semblent pas dépasser l'embouchure de la Gironde. Ce sont :

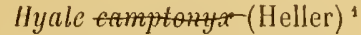

Ampelisca rubella Costa

Eusiroides Della Vallei nov. nom.
Dexamine spiniventris (Costa) Microlleutopus Stationis Della Valle Amphithoe Vaillanti Lucas

D'autre part, les huit espèces suivantes, babitant la côte française de la Manche, n'ont pas encore été rencontrées sur nos côtes de l'Océan :

Metopu Alderi (Sp. Bate)

Paramphithoe bicuspis (Kröyer)

Gammarus campylops Leach

Cheirocratus assimilis Lillj.
Podoceropsis excavata (Sp. Bate)

Corophium crassicorne Bruz.

Dulichia porrecta Sp. Bate

Caprella linearis (Linné) 



\title{
ASSOCIATION FRANÇAISE \\ POUR L'AVANCEMENT DES SCIENCES
}

\section{Extrait des Statuts et Règlement}

\begin{abstract}
STATUTS
Art. 4. - Les membres de l'Association sont admis, sur leur demande, par le Conseil.

ART. 5. - Sont membres de l'Association les personnes qui versent la cotisation amnuelle. Cette cotisation peut toujour's être rachetée par une somme versee une fois pour toutes. Le taux de la cotisation et celui du rachat sont fixés par le Règlement.

ART. 6. - Sont membres fondateurs les personnes qui ont versé, à une époque quelconque, une ou plusieurs souscriptions de 500 franes.

Anт. 7. - Tous les membres jouissent des mêmes droits. Toutefois, les noms des membres fondateurs figurent perpétuellement en tète des listes alphabétiques, et ces membies recoivent gratuitement, pendant toute leur vie, autant d'exemplaires des publications de l'Association qu'ils ont versé de fois la souscription de 500 franes.
\end{abstract}

\section{RĖGLEMENT}

Article premier. - Le taux de la colisation annuelle des membres non fondateurs est fixé à 20 francs.

ART. 2. - Tout membre a le droit de racheter ses cotisations à venir en versant, une fois pour toutes, la somme de 200 franes. 11 devient ainsi membre à vie.

Il sera loisible de racheter les cotisations par deux versements annuels consécutifs de 100 franes.

Les membres ayant priyé pendant 20 années consécutives la cotisation annuelle de 20 francs pourront racheter les colisations à venir moyennant un seul versement de 100 francs.

Tout membre qui pendant 10 années consécutires aura versé annnuellement une somme de 10 franes en sus de la cotisation annuelle sera libẻré de tout versement ultérieur.

La liste alphabétique des membres à vic est publiée en tête de chaque volume, immédiatement après la liste des membres fondateurs.

Les membres ayant rachelé leurs cotisations pourront devenir membres fondateurs en versant une somme complémentaire de 300 francs.

Les souscriptions des membres fondateurs peuvent être versées en une seule fois ou en deux versements annuels consécutifs de 250 trancs.

Les souscriptions sont reçues:

Au Secrétariat, 28, rue Serpente, à Paris. 



